Editorial

\title{
Desde los métodos cualitativos hacia los modelos mixtos: tendencia actual de investigación en ciencias sociales
}

\section{From qualitative methods to mixed models: current trend in social science research}

\author{
M Cruz Sánchez-Gómez ${ }^{1}$, Ana Isabel Rodrigues ${ }^{2}$ e António Pedro Costa ${ }^{3}$ \\ mesago@usal.es, ana.rodrigues@ipbeja.pt, apcosta@ua.pt
}

M Cruz Sánchez-Gómez, e Ana Isabel Rodrigues², António Pedro Costa³

${ }^{1}$ Departamento de Didáctica, Organización y Métodos de Investigación, Facultad Educación, Universidad de Salamanca, España

${ }^{2}$ Departamento de Ciências Empresariais, Instituto Politécnico de Beja, Portugal

${ }^{3}$ Ludomedia/webQDA e CIDTFF/UA - Centro de Investigação Didática e Tecnologia na Formação de Formadores, Departamento de Educação e Psicologia, Universidade de Aveiro, Aveiro, Portugal

DOI: 10.17013/risti.28.o

\section{Introducción}

La metodología hace referencia al modo en que enfocamos los problemas y buscamos las respuestas. Conlleva la reflexión sobre cómo obtener conocimiento, qué se debe hacer y cómo realizarlo. Los propósitos, supuestos e intereses determinan la metodología a seguir en una investigación. Sin embargo, no siempre existe el necesario consenso sobre cómo realizar el acercamiento metodológico a la realidad.

Desde el punto de vista de los métodos de investigación, los cuantitativos y cualitativos, tienen bien definidas sus reglas dejuego, pero existen escasos procedimientos metodológicos que integren realmente estrategias cualitativas y cuantitativas en un mismo estudio. Para los investigadores Mertens, Bazeley, Bowleg, Fielding, Maxwell, Molina-Azorin \& al. (2016) los primeros debates sobre los métodos de las ciencias sociales se remontan al siglo XVII y el uso combinado de métodos cuantitativos y cualitativos se remonta a una fecha más temprana. A lo largo del siglo $\mathrm{XX}$, investigadores de distintas disciplinas y países 
adoptaron enfoques mixtos y multimétodos (Jahoda, Lazarsfeld, \& Zeisel, 1933; Platt, 1996) y especialmente a finales del XX y XXI, han abogado por el uso de métodos mixtos (Anguera, Blanco-Villaseñor, Losada, Sánchez-Algarra \& Onwuegbuzie, 2018).

Los investigadores en la actualidad, motivados en un clima de financiación cada vez más competitivo para fundamentar sus estudios como metodológicamente novedosos, exhaustivos o innovadores, consideran conveniente investigar con modelos mixtos (MM).

En el seminario de trabajo organizado por de la Asociación Internacional de Invetigación de Métodos Mixtos (MMIRA) y otros investigadores (https://mmira.wildapricot. org/) en la Universidad de Gallaudet, en EE. UU. (Mertens et al., 2016), se pone de manifiesto que la investigación con estos métodos se ha ampliado en las últimas décadas con abundantes publicaciones que hacen vislumbrar un desarrollo prometedor de los mismos. Afirman que los métodos mixtos ofrecen lo que denominan "eficiencia teórica", es decir, posibilitan desarrollar un análisis holístico, multidimensional y robusto de los fenómenos sociales más eficaz que con un único método. Implican tanto beneficios técnicos ("ventajas de las tecnologías") como teóricos (analíticos de distintos métodos).

A pesar de las diversas opiniones y de los numerosos debates, existe un consenso creciente de que, al igual que los métodos cuantitativos y cualitativos, la investigación con métodos mixtos, supone un enfoque metodológico distinto (Johnson, Onwuegbuzie \& Turner, 2007). Estos autores parten de la idea de que el uso de enfoques cuantitativos y cualitativos, en combinación, proporciona una mejor comprensión de los problemas de la investigación que cualquier enfoque por separado, y que los modelos mixtos constituyen por si solos una metodología.

En este sentido, MMIRA da importancia a la aparición de nuevos instrumentos tecnológicos en los modelos mixtos, que implican la intersección entre el análisis de datos cualitativos basados en códigos y el análisis de contenido; la convergencia de técnicas de visualización de datos cuantitativos y cualitativos; y el análisis espacial y social. Los investigadores que utilizan métodos mixtos han adoptado con entusiasmo el software cualitativo (“CAQDAS"), que ha desempeñado un papel importante en el análisis más sistemático de los datos cualitativos, y en su integración con los datos cuantitativos (Bazeley, 2010; Silver \& Lewins, 2014). El CAQDAS contemporáneo ofrece cada vez más herramientas poderosas para la semi-automatización del análisis basado en código (Franzosi, Doyle, Mclellan \& al., 2013). Las herramientas digitales para el análisis de contenido han permitido nuevos enfoques analíticos para conjuntos de datos mixtos (Hogenraad, Mckenziey \& Peladeau, 2003; Popping, 2009), incluyendo la automatización completa (Grimmer \& Stewart, 2013) y la minería de textos (Lee, Jong \& Kim, 2010), el reto es mantener la cercanía a los datos subyacentes.

Como se señala en párrafos anteriores, la realidad social es compleja, multivariada y difícil de comprender. El estudio de cada objeto puede beneficiarse de la adopción de distintas metodologías y diseños de investigación, lo que implica una pluralidad metodológica que diversifica los modos de aproximación, descubrimiento y justificación en atención a la faceta o dimensión de la realidad social que pretende estudiar (SánchezGómez, Martín-Cilleros \& Canal Bedia, 2018).

En esta línea de exhaustividad e innovación metodológica se celebró el CIAIQ2018 que tuvo lugar del 10 al 13 de julio de 2018 en la Universidad de Fortaleza, Brasil. 
Fruto de las reflexiones metodológicas, el congreso recibió un total de 443 artículos. Cada uno fue sometido a un proceso de revisión doble ciego, por una comisión científica compuesta por miembros altamente calificados en las áreas científicas del congresso. Esta edición especial de RISTI está compuesta por 8 artículos que basados estas nuevas herramientas digitales para el análisis de contenido, como los CAQDAS, han permitido nuevos enfoques analíticos en estudios multimetodológicos y modelos mixtos, acorde con las tendencias actuales.

\section{Estructura}

El primer artículo identifica el papel de la familia en la construcción de la competencia digital de niños/as de doce años, en hogares con distinto capital cultural, a través de un estudio de casos financiado por el Ministerio de Economía y Competitividad español. Los hallazgos encontrados ponen de manifiesto el distinto significado social que las tecnologías pueden tener en el se no de la familia, y como éstas no son en ningún caso neutrales.

El segundo artículo aborda lo tema del tratamiento de Robótica educativa en Educación Infantil que tien implícita la iniciación del pensamiento computacional y el apoyo al desarrollo de nociones espaciales básicas (delante, detrás, arriba, abajo, derecha e izquierda). El artículo, através de una propuesta didáctica desarrollada en el aula se pretende conocer las diferencias sobre la adquisición de estos conceptos espaciales básicos antes y después de la intervención con el Robot Roamer.

El tercer artículo presenta la tecnología educativa para mediar prácticas educativas sobre alimentación complementaria en la Amazonia. Para ello se valida un manual para mediar la enseñanza-aprendizaje de madres de niños de seis a doce meses sobre la alimentación complementaria. El estúdio se basa en una investigación mixta, realizada en la Universidad del Estado de Pará Campus XIII, en el municipio de Tucuruí-PA. La tecnología educativa fue considerada válida para ser utilizada por los profesionales de la salud con el público objetivo.

El cuarto artículo tiene como objetivo analizar la percepción de los graduandos sobre el proceso de aprendizaje en la Etapa Supervisada Salud Colectiva e la Facultad de Ceilândia-Universidad de Brasilia para constitución de Consejo Gestor de Unidad de Salud con uso de Tecnología Social. La metodología utilizada ha sido una investigaciónacción y análisis de contenido en un corpus extraído del informe y de la evaluación, basada en principios de la metodología activa de enseñanza-aprendizaje y en la Teoría del Agir Comunicativo.

El quinto artículo aborda la política y lo político en Twitter, donde se analizan discursos de los candidatos a la presidencia de Colombia (2018), que tienen lugar en Twitter, demostrando la manera en que los mismos configuran la construcción de una dimensión política. Tomando como base un estudio netnográfico, se interpreta un corpus documental de tweets, emitidos por cuatro candidatos presidenciales, clasificados a partir de cinco categorías de análisis (Corrupción, educación, salud, desempleo y paz). El artículo concluye que las nuevas redes sociales virtuales, operan como espacios de debate, fundamentales para entender los procesos electorales contemporáneos. 
El sexto artículo tiene por objetivo investigar cómo la gestión en CallCenter influye en la precarización del trabajo de los operadores. En lo que se refiere a la metodología, este trabajo se constituye como una investigación descriptiva de naturaleza cualitativa. Como instrumento de recolección de datos, se utilizó un itinerario semiestructurado para la realización de las entrevistas, con operadores de telemarketing en la ciudad del Salvador, en el estado de Bahía. Como resultado se verificó que los operadores de telemarketing tienen su trabajo aún más precarizado en función de la adopción del modelo de gestión en los CallCenters.

El séptimo artículo tiene el propósito de comprender la relación teoría-práctica en la construcción de los saberes docentes. Para ello se realizó un estudio que involucró los cursos de Licenciatura en Física ofrecidos por instituciones públicas catarinenses (Brasil). Se presentan las dimensiones (curricular, pedagógica y práctica) y categorías de análisis de la investigación. Con el cruzamiento de los datos y apoyo del software webQDA fue posible identificar los temas que se tratan en las disciplinas pedagógicas de física y su relación con la construcción del conocimiento pedagógico de contenido de los licenciandos.

Finalmente, el último artículo con el objetivo de conocer la evolución de los diferentes métodos de investigación en el área de las Ciencia Sociales, se realiza un análisis de la producción científica, utilizando para ello dos bases de datos internacionales, web of science y scopus. Se ha seleccionado un periodo de los últimos veinticinco años analizando los progresos tanto en la literatura científica general como en las ciencias sociales específicamente. En este estudio se observa una creciente evolución de los métodos cualitativos y mixtos, así como un ligero descenso de la productividad en general en el último año.

\section{Agradecimientos}

Los editores quisieran finalizar agradeciendo a todos los que de forma directa o indirecta colaboraron con el éxito del CIAIQ2018 y con la producción de este número especial, incluyendo a los participantes, autores, comisión organizadora y científica, apoyos, equipo editorial, entre muchos otros. A través de su interés, participación y calidad y rigor de su trabajo científico, ahora publicado en la RISTI, esperamos que pueda promoverse la expansión de la investigación cualitativa en un área tan relevante como es la de los Sistemas y Tecnologías de Información.

\section{Referencias}

Anguera, M. T., Blanco-Villaseñor, A., Losada, J. L., Sánchez-Algarra, P., \& Onwuegbuzie, A. J. (2018). Revisiting the difference between mixed methods and multimethods: Is it all in the name? Quality \& Quantity, 1-14.

Bazeley, P. (2010). Computer assisted integration of mixed methods data sources and analyses. En: A. Tashakkori, \& C. Teddlie, (Eds.), Handbook of mixed methods in social and behavioral research (2nd ed). Thousand Oaks, CA: Sage. 
Franzosi, R., Doyle, S., McClelland, L.E., Putnam Rankin, C., \& Vicari, S. (2013). Quantitative narrative analysis software options compared: PC-ACE and CAQDAS (ATLAS.ti, MAXqda, and NVivo). Quality \& Quantity, 47(6), 3219-3247.

Grimmer, J., \& Stewart, B.M. (2013). Text as data: The promise and pitfalls of automatic content analysis methods for political texts. Political Analysis, 21(3), 267-297.

Hogenraad, R., Mckenzie, D., \& Peladeau, N. (2003). Force and influence in content analysis: The production of new social knowledge. Quality and Quantity, 37(3), 221-238.

Jahoda, M., Lazarsfeld, P., \& Zeisel, H. (1933). Die Arbeitslosen von Marienthal. English translation published in 1971, as Marienthal: The sociography of an unemployed community. Chicago, IL: Aldine Atherton.

Johnson, R. B., Onwuegbuzie, A. J., \& Turner, L. A. (2007). Toward a definition of mixed methods research. Journal of Mixed Methods Research, 1(2), 112-133.

Lee, S., Song, J., \& Kim, Y. (2010). An empirical comparison of four text mining methods. Journal of Computer Information Systems, 51(1), 1-10.

Mertens, D., Bazeley, P., Bowleg, L., Fielding, N., Maxwell, J., Molina-Azorin, J. F., et al. (2016). The future of mixed methods: A five year projection to 2020. From http://mmira.wildapricot.org/resources/Documents/MMIRA\%20task\%2oforce \%20report\%20Jan2016\%20final.pdf

Mertens, D.M. (2015). Mixed methods and wicked problems. Journal of Mixed Methods Research, 9(1), 3-6.

Platt, J. (1996). A history of social research methods in America, 1920-196o. Cambridge, England: Cambridge University Press.

Popping, R., \& Roberts, C.W. (2009). Coding issues in semantic text analysis. Field Methods, 21(3), 244-264.

Sánchez-Gómez, M. C., Martín Cilleros, M. V., \& Canal Bedia, R. (2018). Sistematización del contenido de entrevistas grupales en las Ciencias de la Salud. Campo Abierto. Revista de Educación, 37 (1), 119-132. ISSN 0213-9529

Silver, C., \& Lewins, A. (2014). Using Software in Qualitative Research: A Step-by-Step Guide (2a ed.). London: Sage (DOI: http://dx.doi.org/10.4135/9781473906907). 